\title{
Multi-spacecraft study of the solar wind at solar minimum: dependence on latitude and transient outflows
}

\author{
R. Laker ${ }^{1 \star}$, T. S. Horbury ${ }^{1}$, S. D. Bale ${ }^{1,2,3}$, L. Matteini ${ }^{1}$, T. Woolley ${ }^{1}$, L. D. Woodham ${ }^{1}$, J. E. Stawarz ${ }^{1}$, E. E. Davies ${ }^{1}$, \\ J. P. Eastwood ${ }^{1}$, M. J. Owens ${ }^{4}$, H. O’Brien ${ }^{1}$, V. Evans ${ }^{1}$, V. Angelini ${ }^{1}$, I. Richter ${ }^{5}$, D. Heyner ${ }^{5}$, C. J. Owen ${ }^{6}$, P. Louarn ${ }^{7}$, \\ and A. Federov ${ }^{7}$
}

\author{
1 Imperial College London, South Kensington Campus, London, SW7 2AZ, UK \\ 2 Physics Department, University of California, Berkeley, CA 94720-7300, USA \\ 3 Space Sciences Laboratory, University of California, Berkeley, CA 94720-7450, USA \\ 4 Department of Meteorology, University of Reading, Earley Gate, PO Box 243, RG6 6BB Reading, UK \\ 5 Technical University of Braunschweig, Braunschweig, Germany \\ 6 Mullard Space Science Laboratory, University College London, Holmbury St. Mary, Dorking, Surrey RH5 6NT, UK \\ 7 Institut de Recherche en Astrophysique et Planétologie, 9, Avenue du Colonel ROCHE, BP 4346, 31028 Toulouse Cedex 4, France
}

Received XXXX; accepted YYYY

\begin{abstract}
Context. The recent launches of Parker Solar Probe (PSP), Solar Orbiter (SO) and BepiColombo, along with several older spacecraft, have provided the opportunity to study the solar wind at multiple latitudes and distances from the Sun simultaneously.

Aims. We take advantage of this unique spacecraft constellation, along with low solar activity across two solar rotations between May and July 2020, to investigate how the solar wind structure, including the Heliospheric Current Sheet (HCS), varies with latitude.

Methods. We visualise the sector structure of the inner heliosphere by ballistically mapping the polarity and solar wind speed from several spacecraft onto the Sun's source surface. We then assess the HCS morphology and orientation with the in situ data and compare with a predicted HCS shape.

Results. We resolve ripples in the HCS on scales of a few degrees in longitude and latitude, finding that the local orientation of sector boundaries were broadly consistent with the shape of the HCS but were steepened with respect to a modelled HCS at the Sun. We investigate how several CIRs varied with latitude, finding evidence for the compression region affecting slow solar wind outside the latitude extent of the faster stream. We also identified several transient structures associated with HCS crossings, and speculate that one such transient may have disrupted the local HCS orientation up to five days after its passage.

Conclusions. We have shown that the solar wind structure varies significantly with latitude, with this constellation providing context for solar wind measurements that would not be possible with a single spacecraft. These measurements provide an accurate representation of the solar wind within $\pm 10^{\circ}$ latitude, which could be used as a more rigorous constraint on solar wind models and space weather predictions. In the future, this range of latitudes will increase as SO's orbit becomes more inclined.
\end{abstract}

Key words. Sun: solar wind - Sun: heliosphere - Sun: coronal mass ejections (CMEs)

\section{Introduction}

Early spacecraft measurements in the ecliptic plane revealed that although the magnetic field was aligned with the Parker spiral (Parker 1958), it would reverse direction several times per solar rotation, either pointed away (positive polarity) or towards (negative polarity) the Sun (Wilcox \& Ness 1965). This, along with a single polarity effect observed out of the ecliptic plane (Rosenberg \& Coleman 1969), led to the idea of a warped Heliospheric Current Sheet (HCS), that extends throughout the heliosphere and separates opposing magnetic polarities (Alfvén 1977; Smith 2001).

At solar minimum, when the Sun's magnetic field can be well described by a dipole configuration, the HCS has a limited latitudinal extent, due to its relation to the tips of closed field lines in the equatorial streamer belt (Gosling et al. 1981; Hoeksema et al. 1983). The HCS also exhibits a low local inclination, meaning that all parts of the HCS are relatively parallel with the

\footnotetext{
* Corresponding author: Ronan Laker e-mail: ronan.laker15@imperial.ac.uk
}

solar equator (Smith \& Thomas 1986; Peng et al. 2017). Therefore, at solar minimum the HCS is generally flat with a well defined shape that can persist for several solar rotations (Thomas \& Smith 1981; Riley et al. 2002). However, at solar maximum, where the magnetic field at the poles is no longer dominant, the HCS covers a wider range of latitudes accompanied by a higher local inclination (Suess et al. 1993; Owens \& Lockwood 2012). This results in a much more complex HCS structure, where there is no longer a single polarity per hemisphere (Hoeksema 1991).

The location and shape of the HCS has a direct impact on the sector polarity and solar wind conditions seen by a spacecraft in the solar wind. Therefore, by modelling the location of the HCS, contextual information about the source region of the solar wind can be gained. This can be achieved by tracking the location of the streamer belt in white light images (Wang et al. 1997; Robbrecht \& Wang 2012; Rouillard et al. 2020), or by using a numerical model driven by remote sensing observations (Odstrcil 2003). The widely adopted potential field source surface (PFSS) model assumes a current-free corona and radial solar wind expansion past a spherical source surface (Schatten 
et al. 1969; Altschuler \& Newkirk 1969). Despite its simplicity, PFSS models have been shown to compare well with more sophisticated numerical models (Riley et al. 2006), as well as in situ measurements at $1 \mathrm{AU}$ (Jian et al. 2015) and closer to the Sun (Badman et al. 2020; Panasenco et al. 2020). The local inclination of the HCS predicted by the PFSS model has also been shown to be consistent with in situ measurements except in those cases where transients, such as coronal mass ejections (CMEs), are present (Klein \& Burlaga 1980; Burton et al. 1994; Peng et al. 2017). In situ manifestations of CMEs are commonly observed around HCS crossings where it has been argued that they carry the sector boundary, rather than being structures that drape or push the HCS aside (Gosling et al. 1987; Crooker et al. 1993; Crooker \& Intriligator 1996; Forsyth et al. 1997). While transients can disrupt the local HCS structure, it is generally accepted that the HCS reforms to its original state, although the timescale of this process is debated (Zhao \& Hoeksema 1996; Crooker et al. 1998; Blanco et al. 2011).

During solar minimum, the solar wind mirrors the magnetic structure of the Sun, with fast solar wind $\left(>600 \mathrm{~km} \mathrm{~s}^{-1}\right)$ emanating from regions of open field at the Sun's poles, known as coronal holes (CHs, McComas et al. 1998), and a slower, more variable, solar wind surrounding the HCS at low latitudes (Zhao \& Hundhausen 1981; Gosling et al. 1981; Pizzo 1994). As a result of coronal structure, solar wind of varying speeds can exist at the same heliographic latitude, which can create a co-rotating interaction region (CIR), providing that the solar wind sources are time stationary (Smith \& Wolfe 1976). A typical CIR consists of a rarefaction at the trailing edge of the fast stream, and a compression region at the leading edge, which envelops the HCS as the CIR develops (Gosling \& Pizzo 1999). CIRs also have a distinctive east-west flow deflection across the stream boundary due to the Sun's rotation (Richardson 2018), although the meridional flow deflections depend on the CIR tilt (Siscoe et al. 1969). This effect has been observed at latitudes $>30^{\circ}$ with Ulysses (Gosling et al. 1993), and have been shown to significantly affect the structure of the HCS (Pizzo 1994; Lee 2000). The compression at the leading edge of a CIR can produce a planar magnetic structure (PMS, Nakagawa et al. 1989), where the local magnetic field is forced to lie in the same plane as the stream interface (Broiles et al. 2012). If these magnetic field deflections have a significant southward component then this, along with increased density and speed, can drive space weather effects at Earth (Tsurutani et al. 2006). Therefore, it is important to understand how CIRs vary with both distance and latitude.

Due to the restriction of single point measurements, many studies have relied on large statistics to investigate CIR properties and development (Richter \& Luttrell 1986; Jian et al. 2006). However, with the recent launches of Solar Orbiter (SO, Müller et al. 2013), Parker Solar Probe (PSP, Fox et al. 2016) and BepiColombo (Benkhoff et al. 2010), there are now an unprecedented number of active spacecraft in the inner heliosphere. These, along with the other missions such as Wind (Ogilvie \& Desch 1997) and the Solar Terrestrial Relations Observatory (STEREO, Kaiser et al. 2008), provide a constellation of spacecraft that can be used collectively to improve upon single spacecraft measurements. Recently, several studies have investigated how certain solar wind features evolve from the close proximity PSP measurements out to $1 \mathrm{AU}$ (Szabo et al. 2020; Panasenco et al. 2020; Allen et al. 2021). In this paper, we demonstrate that this spacecraft configuration can be used to investigate how individual features in the solar wind vary with latitude and distance from the Sun. To visualise the solar wind's sector structure, we ballistically map in situ data from the available spacecraft onto the
Sun's source surface, as outlined in Section 2. We then empirically determine the HCS shape, and compare it to a PFSS model in Section 3.1, while also demonstrating that this technique can identify coherent structures measured by several spacecraft at a range of latitudes (Sections 3.2 and 3.3). In Section 3.4, we present observations of several transient structures and examine their effect on the associated sector boundaries. Finally, our conclusions are presented in Section 4.

\section{Methods}

In this paper, we consider two solar rotations with low solar activity, CR2231 and CR2232, which span from 21 May to 15 July 2020. We refer to the position of the spacecraft in Carrington coordinates, specifically the 'IAU_SUN' frame in NAIF's SPICE framework (Acton et al. 2018). This frame rotates with the Sun's surface, at a rate of $14.18^{\circ}$ day (Archinal et al. 2011), allowing each point on the Sun to be described by a longitude, $\Phi$, and latitude, $\Theta$, which can be extended into the heliosphere by including the distance from the Sun's surface, $R$. We note that the solar equator is not aligned with the ecliptic plane, meaning that the Carrington latitude of a spacecraft orbiting in the ecliptic plane will depend on its Carrington longitude.

\begin{tabular}{|c|c|c|c|}
\hline Spacecraft & Instrument & Type & Reference \\
\hline PSP & FIELDS & Mag & Bale et al. (2016) \\
\hline PSP & SPC & Plasma & $\begin{array}{l}\text { Kasper et al. (2016), } \\
\text { Case et al. (2020) }\end{array}$ \\
\hline PSP & SPAN-e & $e^{-} \mathrm{PAD}$ & $\begin{array}{l}\text { Kasper et al. (2016), } \\
\text { Whittlesey et al. (2020) }\end{array}$ \\
\hline SO & MAG & Mag & Horbury et al. (2020) \\
\hline SO & PAS & Plasma & Owen et al. (2020) \\
\hline BepiColombo & MAG & Mag & $\begin{array}{l}\text { Glassmeier et al. (2010) } \\
\text { Heyner et al. (2020) }\end{array}$ \\
\hline STEREO-A & IMPACT & Mag & Acuña et al. (2008) \\
\hline STEREO-A & PLASTIC & Plasma & Galvin et al. (2008) \\
\hline Wind & MFI & Mag & Lepping et al. (1995) \\
\hline Wind & SWE & Plasma & Ogilvie et al. (1995) \\
\hline Wind & $3 \mathrm{DP}$ & $e^{-} \mathrm{PAD}$ & Lin et al. (1995) \\
\hline
\end{tabular}

Table 1. List of the different measurements used in this paper, for the period spanning 21 May to 15 July 2020. All spacecraft had magnetic field (Mag) data available, although only Wind and PSP had bulk plasma parameters and electron pitch angle distributions (PADs).

We used data from a wide variety of spacecraft throughout the inner heliosphere, where a full list of each available dataset can be found in Table 1. PSP reached a perihelion of $0.13 \mathrm{AU}$ on the 7th June 2020 during its fifth solar encounter and continued taking data out to $\sim 0.5$ AU. As seen in Table 1, both PSP and Wind provided all three types of data used in this paper: magnetic field, bulk proton parameters and the electron strahl. This was not the case for SO, which was launched in February 2020, and was in the commissioning phase during this paper's period of interest. Therefore, there are only a few days of bulk plasma data from the Proton-Alpha Sensor (PAS), in early June when SO was predicted to encounter the tail of comet ATLAS (Jones et al. 2020). However, the MAG instrument continuously measured the magnetic field throughout these two solar rotations, at distances ranging from $0.51 \mathrm{AU}$ at perihelion to $0.63 \mathrm{AU}$.

The BepiColombo spacecraft was in the cruise phase ahead of orbiting Mercury at the end of 2025 (Steiger et al. 2020), and had completed an Earth flyby on the 10 April 2020. Therefore, 
BepiColombo was close to Earth during this period, with a radial distance from the Sun decreasing from 0.99 AU to 0.85 AU. The magnetometer (MAG) aboard the Mercury Planetary Orbiter (MPO) was operating at 16 vector/second. This data has been ground calibrated regarding temperature dependence of sensitivity, misalignment and sensor offset. Furthermore, a quasi-static spacecraft disturbance field, derived from long term in-flight experience, is removed, but any time dependent disturbances are still visible in the data. However, we mitigated this issue by assessing the data for artificial signals and only considering an average magnetic field over several hours to investigate the polarity and relative magnetic field strength.

We estimated the shape of the HCS and the distribution of open field lines by implementing the PFSS model using the open source pfsspy Python package (Yeates 2018; Stansby et al. 2020b). We supplied a synoptic magnetogram from the Air Force Data Assimilative Photospheric Flux Transport (ADAPT) model, which attempts to forecast flux transport, allowing for more accurate results outside of the Earth's field of view (Hickmann et al. 2015). The underlying assumptions of the PFSS model are that the corona is current free and the field becomes radial past some arbitrary source surface. Recently, several papers have suggested using a variable source surface height (Badman et al. 2020; Panasenco et al. 2020). However, we opted for a constant source surface height so that all spacecraft measurements could be mapped back to a single reference distance. We use an average of the 12 realisations from a single ADAPT model, with a constant source surface height of 2 solar radii, $R_{\odot}$, which has been shown to provide a better match to the magnetic field polarity than the widely used value of $2.5 R_{\odot}$ (Nikolić 2019; Badman et al. 2020).

We performed this mapping using a simple ballistic propagation (Nolte \& Roelof 1973; Stansby et al. 2019b), which assumes a constant radial solar wind speed, $V_{S W}$. This allows us to compare spacecraft measurements taken at different distances, as well as with the PFSS model. This technique transforms the spacecraft's position at a time $t$, described by $\Phi_{S C}$ and $R_{S C}$, to a longitude at the source surface given by:

$\Phi_{S S}(t)=\Phi_{S C}(t)+\frac{\Omega\left(R_{S C}(t)-2 R_{\odot}\right)}{V_{S W}(t)}$,

where $\Omega$ is the solar rotation rate used by the IAU_SUN frame, and $S_{S C}$ denotes that a property belongs to a spacecraft. Throughout this paper we refer to $\Phi_{S S}$ as longitude, unless otherwise specified. We used a six-hour average to determine $V_{S W}$, where bulk proton data was available (Table 1). There is no change to the latitude as a radial flow is assumed. Since BepiColombo orbited close to Earth we used data from Wind to provide a contextual solar wind speed to be used for the mapping. We assumed a speed of $350 \mathrm{~km} \mathrm{~s}^{-1}$ for the SO spacecraft, based on Wind observations across the two solar rotations. A deviation of $50 \mathrm{~km} \mathrm{~s}^{-1}$ from this assumed speed would have resulted in a $\sim 5^{\circ}$ error in $\Phi_{S S}$ for SO at $\sim 0.5 \mathrm{AU}$. There is also an inherent uncertainty in this ballistic mapping owing to the interaction between different solar wind parcels, that can only be estimated by a more sophisticated model than used here (e.g. Roussev et al. 2003; Owens et al. 2020a).

In an effort to establish the sector structure of the solar wind, we determined the magnetic polarity of each six-hour period used in the mapping. Under normal solar wind conditions, the interplanetary magnetic field lies along the Parker spiral (Parker $1958)$, either pointing towards $\left(\phi_{P S, T}\right)$ or away $\left(\phi_{P S, A}\right)$ from the Sun. We calculated the Parker spiral angle for each six-hour periods, using the same $R_{S C}, V_{S W}$ and $\Omega$ values from the ballistic mapping. We refer to the magnetic field in Radial-TangentialNormal (RTN) coordinates, where $\boldsymbol{R}$ points from the Sun to the spacecraft, $N$ is the component of the solar north direction perpendicular to $\boldsymbol{R}$, and $\boldsymbol{T}$ completes the right-handed set. We express the magnetic field direction as angles in the R-T $(\phi)$, and T-N planes $(\theta)$, where $\phi=0^{\circ}, \theta=0^{\circ}$ is along $\boldsymbol{R}$ and $\phi=90^{\circ}, \theta=0^{\circ}$ is parallel to $\boldsymbol{T}$. We assigned the magnetic polarity as being outwards when $\phi_{P S, A}-45^{\circ}<\phi<\phi_{P S, A}+45^{\circ}$, and inwards when $\phi_{P S, T}-45^{\circ}<\phi<\phi_{P S, T}+45^{\circ}$. If $\phi$ lay outside this range of angles then we assigned the polarity as mixed.

While the magnetic field data allowed us to observe when the magnetic polarity changed, as is expected at a HCS crossing, it does not necessarily mark the location of the sector boundary (Crooker et al. 2010; Owens et al. 2013). Therefore, to investigate the true connectivity of our identified events, we studied the pitch angle distribution (PAD) of the suprathermal electron population, where the pitch angle ranged from $0^{\circ}$ (parallel) to $180^{\circ}$ (anti-parallel) to the local magnetic field direction (Feldman et al. 1975; Pilipp et al. 1987). This population, called strahl, is created in the solar corona (Rosenbauer et al. 1977), so it is expected that the interplanetary magnetic field with (inwards) outwards magnetic polarity will exhibit a (anti-) parallel strahl population. The presence of Bi-Directional Electrons (BDEs), streaming both parallel and anti-parallel, implies that the field is connected to the Sun at both ends (Palmer et al. 1978; Bame et al. 1981). Hence, the strahl PADs were an important diagnostic tool. However, this type of data was only available for the Wind and PSP spacecraft in our period of interest. Therefore, we also calculated the cross helicity, $\sigma_{C}$, of the solar wind in 30 min intervals (Bruno \& Carbone 2013; Stansby et al. 2019a). The magnitude of $\sigma_{C}$ indicates the degree at which there are unidirectional Alfvénic fluctuations within a given period, where $\left|\sigma_{C}\right| \leq 1$. The sign of $\sigma_{C}$ indicates the direction of travel for the fluctuations with respect to the magnetic field, with negative (positive) values indicating outward (inward) polarity. Since Alfvénic fluctuations dominantly travel away from the Sun in the plasma frame, $\sigma_{C}$ can be used as a proxy for magnetic polarity.

After assessing the connectivity of a sector boundary, we applied Minimum Variance Analysis (MVA), taking the orientation of the HCS as the plane normal to the minimum variance direction. Similar to Burton et al. (1994), we consider the whole sector boundary period, rather than analysing individual directional discontinuities, since Klein \& Burlaga (1980) found that these were not a reliable indicator of the overall sector boundary orientation. For each sector boundary, we applied MVA to a window of duration, $\mathrm{T}$, that was shifted across the event in 10 minute steps. To ensure the quality of MVA over a given window, it was required that the ratio of the intermediate to minimum eigenvalues, $\lambda_{2} / \lambda_{3} \geq 5$, along with the value of $\left|B_{n}\right| /|\boldsymbol{B}|<0.2$ (Jones \& Balogh 2000; Kilpua et al. 2017), where $B_{n}$ is the magnetic field component along the minimum variance direction. We used the results from the longest duration window with the largest value of $\lambda_{2} / \lambda_{3}$, which can be found in Table A.1.

In an attempt to distinguish between transient and co-rotating structures, we have compiled a catalogue of events during our period of interest (Table A.1). To make referencing events more straightforward, we have assigned each event a unique identifier, for example 'SO_2005XN'. 


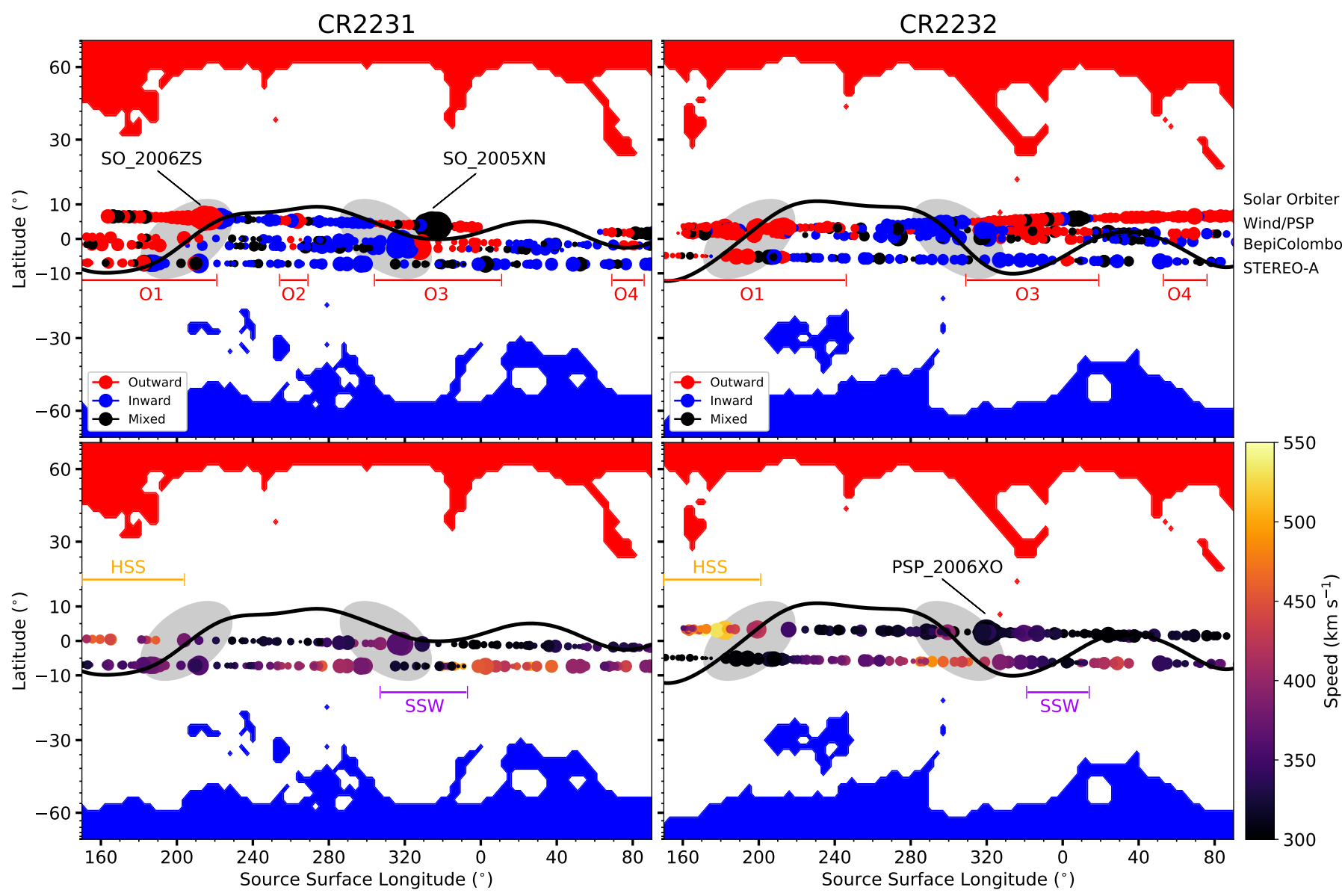

Fig. 1. Each scatter point represents a 6-hour average of the in situ parameters from a single spacecraft, where the size of the scatter point is proportional to $\left(|\boldsymbol{B}| \times R_{S C}\right)^{2}$ to accentuate changes in $|\boldsymbol{B}|$. The top panels show the polarity measured by the different spacecraft for the two solar rotations, as outlined in Section 2, with the order of the spacecraft trails next to the top right panel. In these plots, each spacecraft travelled from right to left, where measurements of the same longitude were made by all the spacecraft within a few days, therefore minimising temporal effects. Open field lines are shown as the colourmap, as calculated from a PFSS model using an ADAPT magnetogram from the 1st June and 1st July for rotation CR2231 and CR2232, respectively. We ensured the output of the PFSS model was stable by considering the predicted HCS shape at daily intervals throughout the periods of interest, with the estimated HCS position shown as the solid black line, which broadly matches the shape of the in situ observations. The bottom left panel shows the solar wind speed from Wind (above) and STEREO-A (below), with the bottom right panel also displaying the data from PSP at the same latitude as Wind. These maps, along with the open field lines from PFSS, show that the CH structure was stable over the two rotations with a CIRs shown as highlighted grey regions at $\sim 200^{\circ}$ and $\sim 320^{\circ}$ longitude. This stable structure is also reflected in the polarity measurements, with outward polarity dips in the HCS outlined with the horizontal red lines. Transient structures are labelled with the according identification.

\section{Results and discussion}

\subsection{HCS structure}

We applied the ballistic mapping technique, described in Section 2, to the constellation of spacecraft outlined in Table 1 to produce the polarity and speed maps seen in Fig. 1. This demonstrates a clear variation in magnetic polarity with latitude, which reveals that the HCS structure was remarkably flat across these two solar rotations (within $\pm 10^{\circ}$ latitude). While such a technique has been applied to spacecraft data before (Schwenn et al. 1978; Villante et al. 1979; Burlaga et al. 1981), this constellation provided an unprecedented level of detail in latitude, which was able to resolve several dips in the HCS (regions O1 to O4). The polarity structure was stable across the two solar rotations, supporting the idea of a stable coronal structure, and therefore HCS shape, which was further evidenced by the similarity in the solar wind speed distribution. This can be seen in the bottom axes of Fig. 1 where a high speed stream (HSS) was observed by Wind and PSP at longitudes $<200^{\circ}$ which, along with other speed gradients, led to the formation of several CIRs highlighted in grey.

The estimated HCS position from a PFSS model (black line), generally matches the shape of the HCS from the in situ measurements, and produces similar open field line distributions across the two solar rotations (colourmap in Fig. 1). While we see a consistent shape between the PFSS model and in situ measurements, we do not attempt a more detailed comparison as this would involve more careful application of PFSS, that may require adjusting the source surface height (Badman et al. 2020; Panasenco et al. 2020; Kruse et al. 2021), or using more complex models (Odstrcil 2003; Jian et al. 2015; Pomoell \& Poedts 2018). However, we do note that the number of measurements over this range of latitudes could be used to better constrain the polarity and solar wind speed predicted by solar wind models. This would be most relevant at solar minimum, since Fig 1 shows that a few degrees of latitude can drastically change the solar wind conditions experienced by a spacecraft, which agrees with 

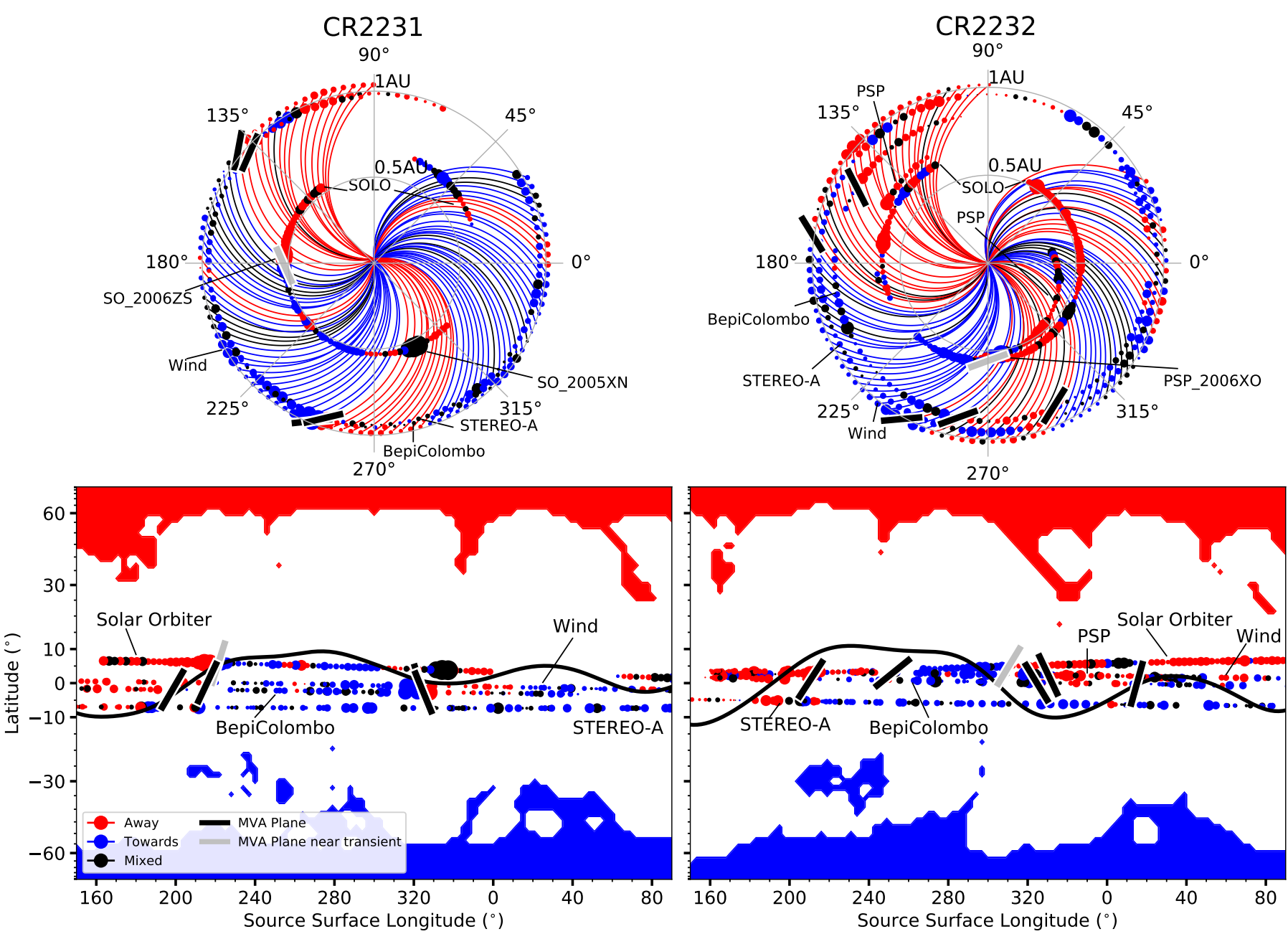

Fig. 2. Top panels show the in situ measurements in the IAU_SUN frame, where the angle of the HCS found from MVA is compared to the Parker spiral. The angles surrounding are of Carrington longitude, which is not the source surface longitude, as plotted in the bottom panels. The source surface longitude can be estimated by following a Parker spiral line which is plotted based on measurements from Wind. These angles are generally parallel to the local Parker spiral direction, with the boundaries associated with transients shown as grey. These plots also give context to where the different spacecraft were during these two rotations, with BepiColombo progressing from 0.99 AU to 0.85 AU. The bottom panels show the source surface as seen in Fig 1, with the in situ orientation from different spacecraft overlain, with the values in Table A.1. Although the orientations generally match the direction expected from the HCS, they are much steeper.

the latitude scale size found by previous studies (Schwenn et al. 1978; Owens et al. 2020b).

The local orientation of the sector boundaries can be seen in Fig. 2, where the Parker spiral lines are drawn from the position of the Wind spacecraft, using the measured solar wind speed. In general, the plane measured from MVA was aligned along the Parker spiral direction in the R-T plane (top panels), and the orientation out the R-T plane (bottom panels) matched the sense of the inclination from the PFSS model. This was also consistent with the shape traced out by several spacecraft crossings of the same sector boundary at different latitudes, best demonstrated with region O1 in CR2231. These results support previous studies (at solar maximum) that found the PFSS model agreed with the in situ orientation, in the absence of any transient structures (Klein \& Burlaga 1980; Burton et al. 1994; Peng et al. 2017). Unlike these studies, we measured the local orientation to be steeper than the relatively flat HCS seen with in situ observations and the PFSS model. We note that this is most likely due to the presence of stream interactions at these boundaries, which steepen with distance from the Sun (Pizzo 1991). Therefore, these observations could represent the start of HCS distortion, that is known to be significant further from the Sun (> 2 AU, Pizzo 1994; Lee 2000; Riley et al. 2002). Although we have also estimated the orientation of boundaries at $0.5 \mathrm{AU}$, they cannot be used as evidence of this steepening argument as they are in close proximity to transient events (shown in grey in Fig. 2) that will be discussed in the Section 3.4.

\subsection{Co-rotating Interaction Regions}

Instead of applying statistics to a large number of CIRs, this spacecraft constellation allows the study of how individual CIRs vary with latitude. One such example is the CIR around $200^{\circ}$ longitude in CR2231, which was measured by four spacecraft, with the time series of Wind and STEREO-A data being shown in Fig. 3. Wind observed the solar wind speed to increase from $300 \mathrm{~km} \mathrm{~s}^{-1}$ to $465 \mathrm{~km} \mathrm{~s}^{-1}$, along with the typical flow deflections expected from a CIR (Fig. 3 panel 5). This HSS originated from the northern polar coronal hole $(\mathrm{CH})$. Therefore, $\mathrm{SO}$, at a higher latitude than Wind, was connected deeper into the $\mathrm{CH}$, which was supported by the presence of large amplitude Alfvénic fluctuations (Belcher \& Davis Jr. 1971). STEREO-A, at $-7^{\circ}$ lati- 
tude, only measured an increase in solar wind speed from 320 $\mathrm{km} \mathrm{s}^{-1}$ to $350 \mathrm{~km} \mathrm{~s}^{-1}$, but recorded a similar $|B|$ profile and increase in proton density. This type of compression, without a clear HSS at similar latitude, has been observed with earlier spacecraft (Burlaga 1983; Schwenn 1990) and is present in the same CIR on the next solar rotation (CR2232 of Fig. 1).

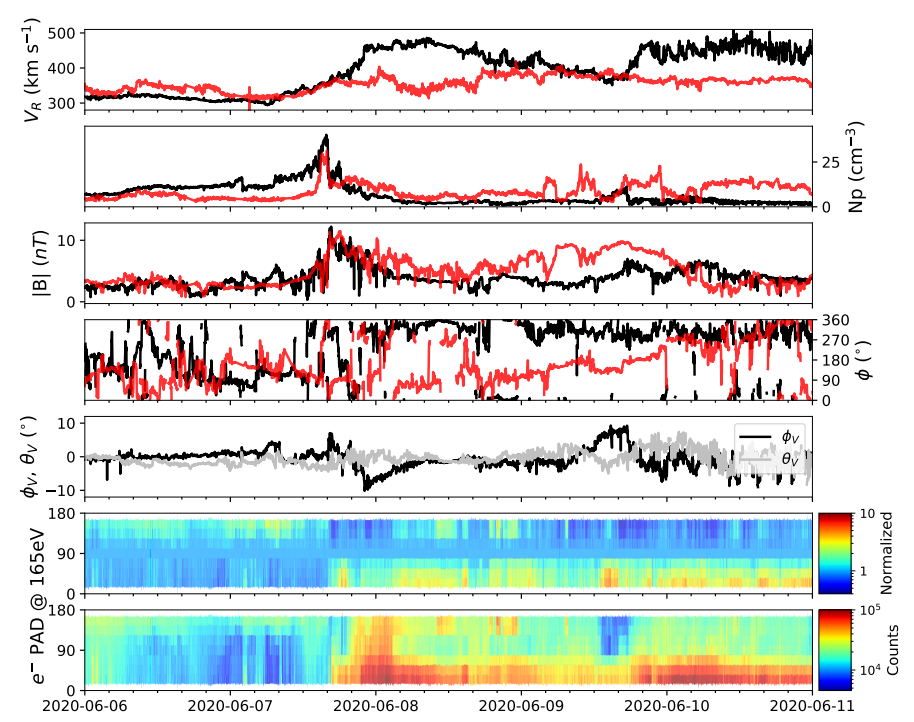

Fig. 3. The CIR observed by Wind and STEREO-A which represents the region $\mathrm{O} 1$ in Fig. 1. Here STEREO-A measurements (red), from the same source surface longitude, have been time shifted by $\sim 6$ days to line up with the increase in $|\mathrm{B}|$. Wind observes a clear increase in solar wind speed from $300 \mathrm{~km} \mathrm{~s}^{-1}$ to $465 \mathrm{~km} \mathrm{~s}^{-1}$, along with a distinctive eastwest (positive-negative) deflection in the $\phi_{V}$ signature. Both spacecraft observe a similar increase in $|\boldsymbol{B}|$ and $N_{p}$, but STEREO-A only measures an increase of $320 \mathrm{~km} \mathrm{~s}^{-1}$ to $350 \mathrm{~km} \mathrm{~s}^{-1}$.

These measurements can be explained by considering the tilt of this particular CIR, as seen in Fig 2. This introduced a southern component to the direction of the forward propagating pressure wave at the leading edge of the CIR, which allowed the HSS to compress slow solar wind beyond its own latitudinal extent. Further from the Sun, this forward propagating compression wave will likely steepen into a forward shock, which have been shown to propagate perpendicular to the stream interaction boundary with the Ulysses spacecraft (Gosling \& Pizzo 1999) and numerical models (Riley et al. 2012). Although this forward shock has not yet developed in these measurements (at 1 AU), we have shown that the solar wind is still affected on scales of a few degrees in latitude by the CIR tilt.

This corroborates the findings from the twin Helios spacecraft (Schwenn et al. 1978), demonstrating the importance of latitude when investigating CIRs, which is also evident in the other CIR $\sim 300^{\circ}$ longitude in Fig. 1. Here STEREO-A measured a gradient solar wind speed, along with an increase in $|\boldsymbol{B}|$ and proton density. However, unlike typical CIR observations, STEREO-A did not measure a change in the magnetic polarity. Therefore, these measurements represent the lower extent in latitude of a CIR without the presence of the HCS, which may alter how the CIR develops. We verified the presence of typical CIR flow deflections in STEREO-A, although the measurements were not reliable enough to carry out a detailed analysis on the CIR dynamics. While we were limited by the lack of plasma and electron measurements from some spacecraft, we have demonstrated how such a multi-spacecraft study can isolate changes in latitude for an individual CIR, which may be important for space weather prediction.

\subsection{Identifying co-rotating structure}

The spread of spacecraft latitude can also provide contextual information, allowing for identification of solar wind structures that would otherwise not be possible. One such example is the small scale dip in the HCS labelled as $\mathrm{O} 2$ in the top left panel of Fig. 1. This outward polarity region was observed by SO, Wind and BepiColombo over a range of $-2.3^{\circ}$ to $5.4^{\circ}$ latitude, and spanned $\sim 15^{\circ}$ longitude in SO. The initial change from inward to outward magnetic field was measured by SO at 16:00 on 3 June 2020 at $0.5 \mathrm{AU}$, which was followed by BepiColombo $\sim 2$ hours later at $1 \mathrm{AU}$, and at Wind a further $~ 13$ hours after. Since BepiColombo and Wind were both orbiting at 1 AU during CR2231 (as seen in Fig 2), their separation in longitude meant that BepiColombo should see a co-rotating structure roughly 12 hours prior to Wind. This, along with the short time interval between BepiColombo and SO, suggests that this field reversal was a co-rotating structure, most likely associated with a ripple in the HCS of similar size to those proposed by Gosling et al. (1981) and Villante et al. (1979). Wind observations of the electron strahl confirmed that this field reversal was related to a sector boundary, with the longer period in SO implying that this was a fine scale ripple in the HCS towards lower latitudes.

There was extra complexity in the SO measurements, with exotic wave activity and an additional inward pointing magnetic field region in the otherwise outward polarity region. This could be due to magnetic field draping and pick-ions related to comet ATLAS, which SO was predicted to interact with (Jones et al. 2020) and is studied in more detail by Matteini et al. (2021). STEREO-A measurements at $-7^{\circ}$ latitude showed no reversal in polarity, and observed the trailing edge of a HSS, unlike Wind measurements of $315 \mathrm{~km} \mathrm{~s}^{-1}$, which implies that this dip does not extend much further south than BepiColombo. This also provided an upper limit of $5^{\circ}$ latitude for the thickness of the streamer belt, which is consistent with earlier observations (Richardson \& Paularena 1997; Chen et al. 2021). Therefore, mapping the speed of the solar wind can further constrain the location of the HCS, rather than relying solely on the magnetic field polarity.

Another example of this is the outward polarity region $\mathrm{O} 3$ which, like O2, did not exhibit a magnetic field reversal in STEREO-A in CR2231. However, in this case STEREO-A did measure slow solar wind (labelled SSW in Fig 1) which confirms that $\mathrm{O} 3$ extended to lower latitudes than $\mathrm{O} 2$. Using the $5^{\circ}$ estimated width of the streamer belt, we conclude that STEREO-A was likely at the edge of the streamer belt. Such a classification could be useful for studying specific situations in the solar wind, like the formation of the Kelvin-Helmholtz instability.

Although the spacecraft used in this paper were not a coordinated effort to create a multi-spacecraft mission, their wide variety of orbits can be used to isolate a particular aspect of solar wind evolution: either time, latitude or distance from the Sun. In the future, these opportunities will be able to exploit different aspects of each spacecraft's orbit, since SO will become more inclined to the ecliptic plane (by around $30^{\circ}$ ) and PSP will measure the solar wind at progressively closer distances to the Sun.

\subsection{Transients}

By comparing measurements from multiple spacecraft, we were also able to classify several transient structures, which are labelled as such in Table A.1. The most complex of these events was SO_2006ZS, labelled in Figs. 1 and 2, which was likely the 

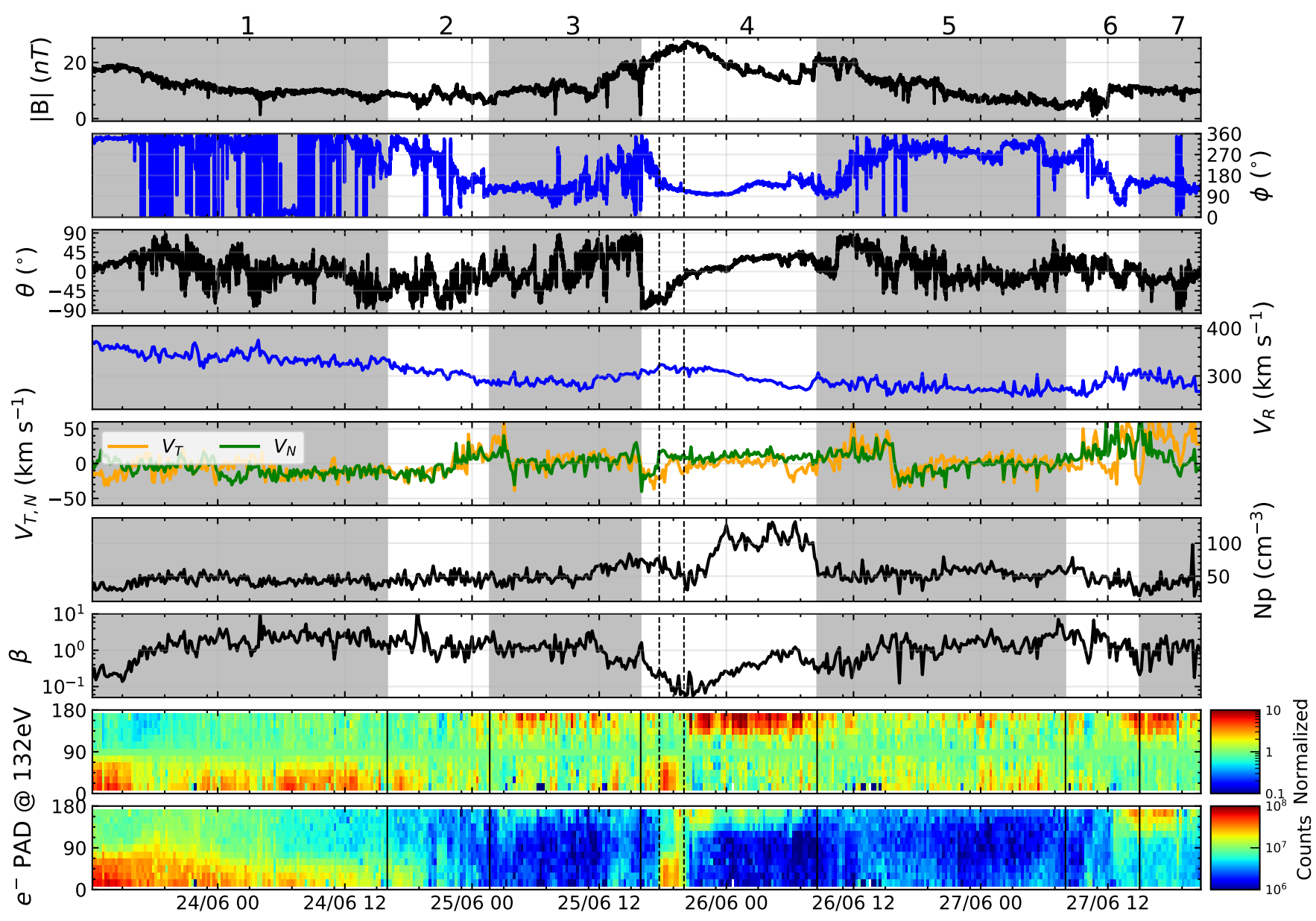

Fig. 4. Timeseries of a sector boundary observed by PSP, with ambient solar wind conditions seen in regions 1 and 7 , and the transient event (PSP_2006XO) appearing in region 4. This was classified as an ICME due to the decreasing radial speed, reduction in $\beta$ and smooth magnetic field rotation. The plasma parameters have been smoothed over 30 minutes. The penultimate panel shows the electron strahl PAD, normalised to its value at $90^{\circ}$. The electron signature, in the bottom two panels, weakens in regions 3 and 5 which could be due to scattering processes.

combination of two CMEs, a CIR and a HCS crossing, which is investigated in detail by Telloni et al. (2021).

A flux rope like structure was observed by SO on 29 May 2020 (SO_2005XN) at $~ 0.5$ AU (Fig A.1). This structure displayed a smooth rotation in the magnetic field with a minimium variance direction along the radial. A similar structure was observed on the following solar rotation at $\sim 0.5$ AU by PSP on 26 June 2020 (PSP_2006XO), as shown in Fig 4. Again, this was a flux rope like structure with an increase in $|\boldsymbol{B}|, \theta$ ranging from $-80^{\circ}$ to $45^{\circ}$ and was confined to a plane with a normal direction along $\boldsymbol{R}$. Like SO_2005XO, this was seen at around $280^{\circ}$ Carrington longitude, which may suggest that these were co-rotating structures. However, we conclude these are interplanetary CMEs (ICMEs) due to absence of these structures in other spacecraft, low plasma $\beta$ and the smoothly decreasing radial speed in region 4 of Fig 4, which is indicative of ICME expansion (Zurbuchen \& Richardson 2006; Richardson \& Cane 2010).

Therefore, these measurements could represent the same type of ICME from the same source region and similar distances from the Sun. While we have identified possible CMEs in remote sensing observations ${ }^{1}$, we leave modelling of these events for a future study and instead focus on the in situ characteristics.

126 May 2020 00:39 for SO_2005XN and 22 June 2020 15:09 for PSP_2006XO in the DONKI catalogue https://kauai.ccmc.gsfc. nasa.gov/DONKI/
The ICME, in region 4 of Fig 4, appears in conjunction with the HCS crossing, which transitions from outward polarity with parallel strahl electrons in region 1 , to inward polarity and antiparallel strahl in region 7. Similar ICMEs have been observed previously, and have been interpreted as magnetic clouds that are part of the HCS, rather than pushing it aside or draping (Crooker et al. 1993; Crooker \& Intriligator 1996; Forsyth et al. 1997).

Within region 4 , there was a period of parallel electrons (bounded by dashed vertical lines) with an inward magnetic field, implying these field lines were folded by the ICME, which could explain why the strahl direction changes when $\theta$ changes sign. The flux rope itself (after the latter dashed line) had antiparallel, rather than counter-streaming electrons, implying it was connected to the Sun at one end (Crooker et al. 2008). This indicates magnetic reconnection has occurred, either during interchange reconnection as it escaped from the Sun (Crooker et al. 1993; Gosling et al. 1995) or as it propagated through the solar wind (McComas et al. 1994). Following the ICME, the magnetic field returned to outward magnetic polarity in region 5, with evidence of weak counter-streaming electrons. This could imply the field lines were connected at both ends to the Sun, although it was more likely due to reflection or focusing of electrons at some boundary in this complex magnetic structure (Gosling et al. 2001).

While Fig 4 displays an ICME within a sector boundary, this same HCS crossing without an ICME was observed by Wind 
and BepiColombo at a similar time, which represented an earlier configuration of the solar wind from the same source region. Wind observed an increase in $|\boldsymbol{B}|$ and solar wind speed, indicating a weak CIR (WIND_2006JJ). The minimum variance normal was along $\left(34^{\circ} \phi, 26^{\circ} \theta\right)$ and $\left(33^{\circ} \phi, 32^{\circ} \theta\right)$ for Wind and BepiColombo respectively. In contrast, SO experienced this HCS crossing $\sim 5$ days after the passage of the ICME in PSP, representing the same source region at a later date and observed the sector boundary plane with a normal along $\left(20^{\circ} \phi,-32^{\circ} \theta\right)$. We propose that this was due to the HCS still reforming after the eruption of a CME that was previously observed by PSP.

While it is well known that the HCS reforms after the passage of a CME (Zhao \& Hoeksema 1996; Blanco et al. 2011), it is unclear over what timescale this process takes place. If these SO measurements do indeed represent a disrupted HCS, then we argue that this puts a lower limit of $\sim 5$ days on this reformation. Similar timescales have been found ( $\sim 3$ to 6 days) in numerical studies that simulated the passage of a CME through background solar wind (Temmer et al. 2017; Desai et al. 2020). However, the lack of plasma and electron measurements at SO make this claim purely speculative, although it does demonstrate the applicability of multi-spacecraft studies on transient solar wind structures.

\section{Conclusions}

After the recent launches of Parker Solar Probe and Solar Orbiter, there are an unprecedented number of spacecraft simultaneously measuring the solar wind. During the period of May to July 2020 these spacecraft, along with BepiColombo, Wind and STEREO-A, provided the opportunity to study the solar wind at a range of latitudes. By mapping the magnetic polarity measured by each spacecraft, we have demonstrated how the structure and position of the HCS can be investigated, finding that:

- The solar wind measured by a spacecraft at solar minimum depended largely on the latitude of the observation.

- The HCS was remarkably flat over two solar rotations (CR2231 and CR2232) between May and July 2020, meaning we were able to resolve fine scale ripples in the HCS down to scales of several degrees in latitude.

- The location of the HCS was further constrained by examining the solar wind speed at each spacecraft, as this could reveal times when a spacecraft was within the slow solar wind surrounding the HCS without changing polarity.

- A PFSS model captured the general shape of the HCS, and agreed with the locally measured sector boundary orientations, but were much steeper. However, due to the presence of transient events we could not observe how the HCS steepened between 0.5 and $1 \mathrm{AU}$.

- A CIR was measured at four different latitudes, which showed that compressed slow solar wind was observed by STEREO-A even in the absence of an accompanying high speed stream. This is evidence for the compression region propagating in latitude.

- Another CIR was observed by STEREO-A without any change in magnetic polarity, highlighting how important latitude is to the CIR structure.

- We could identify several transient structures around sector boundaries, that we classified as ICMEs. By observing a HCS crossing before, during and after an ICME interaction we found evidence of distortion that lasted at least 5 days.

These results highlight that at solar minimum the solar wind varies on scales of just a few degrees in latitude, which can drastically alter the solar wind conditions measured by different spacecraft. Therefore, this combination of spacecraft can be used to constrain solar wind models in latitude, which could improve space weather predictions.

We also note that by comparing measurements across these spacecraft, we can give context to solar wind measurements, that would not otherwise be possible. This could open up new research opportunities since one can be more confident of where a spacecraft is in relation to large scale structures - i.e. crossing the bottom of a CIR, or skimming the HCS.

It is important to note that this collection of spacecraft were not intended to act as a multi-spacecraft mission. Therefore, each spacecraft has its own unique orbital characteristics, with PSP going closer to the Sun; BepiColombo travelling to Mercury and SO increasing the inclination of its orbit. This means that the configuration of the spacecraft will evolve with time, which will continue to provide unique avenues for future heliospheric research.

Acknowledgements. RL was supported by an Imperial College President's Scholarship, TSH and JPE by STFC ST/S000364/1, TW by ST/N504336/1, LDW by ST/S000364/1, CJO by ST/5000240/1. SDB acknowledges the support of the Leverhulme Trust Visiting Professor program. The SWEAP and FIELDS teams acknowledge support from NASA contract NNN06AA01C. The Solar Orbiter magnetometer was funded by the UK Space Agency (grant ST/T001062/1). The Solar Orbiter Solar Wind Analyser (SWA) PAS were designed, created, and are operated under funding provided in numerous contracts from the UK Space Agency (UKSA), the UK Science and Technology Facilities Council (STFC), the Centre National d'Etudes Spatiales (CNES, France), the Centre National de la Recherche Scientifique (CNRS, France), and the Czech contribution to the ESA PRODEX programme. . Solar Orbiter data are available from the Solar Orbiter Archive at http://soar.esac.esa.int/soar/. This work has made use of the open source and free community-developed space physics packages HelioPy (Stansby et al. 2020a), pfsspy (Stansby et al. 2020b) and SpiceyPy (Annex et al. 2020). Solar Orbiter is a space mission of international collaboration between ESA and NASA, operated by ESA.

\section{References}

Acton, C., Bachman, N., Semenov, B., \& Wright, E. 2018, Planetary and Space Science, 150,9

Acuña, M. H., Curtis, D., Scheifele, J. L., et al. 2008, Space Science Reviews, 136,203

Alfvén, H. 1977, Reviews of Geophysics, 15, 271

Allen, R. C., Ho, G. C., Mason, G. M., et al. 2021, Geophysical Research Letters, 48, e2020GL091376

Altschuler, M. D. \& Newkirk, G. 1969, Solar Physics, 9, 131

Annex, A., Pearson, B., Seignovert, B., et al. 2020, AndrewAnnex/SpiceyPy: SpiceyPy 3.1.1

Archinal, B. A., A'Hearn, M. F., Bowell, E., et al. 2011, Celestial Mechanics and Dynamical Astronomy, 109, 101

Badman, S. T., Bale, S. D., Martínez Oliveros, J. C., et al. 2020, The Astrophysical Journal Supplement Series, 246, 23

Bale, S. D., Goetz, K., Harvey, P. R., et al. 2016, Space Science Reviews, 204, 49

Bame, S. J., Asbridge, J. R., Feldman, W. C., Gosling, J. T., \& Zwickl, R. D. 1981, Geophysical Research Letters, 8, 173

Belcher, J. W. \& Davis Jr., L. 1971, Journal of Geophysical Research (18961977), 76, 3534

Benkhoff, J., van Casteren, J., Hayakawa, H., et al. 2010, Planetary and Space Science, 58, 2

Blanco, J. J., Hidalgo, M. A., Rodriguez-Pacheco, J., \& Medina, J. 2011, Journal of Atmospheric and Solar-Terrestrial Physics, 73, 1339

Broiles, T. W., Desai, M. I., \& McComas, D. J. 2012, Journal of Geophysical Research: Space Physics, 117, 1

Bruno, R. \& Carbone, V. 2013, Living Reviews in Solar Physics, 10

Burlaga, L. F. 1983, Journal of Geophysical Research: Space Physics, 88, 6085 Burlaga, L. F., Hundhausen, A. J., \& Zhao, X.-p. 1981, Journal of Geophysical
Research, 86, 8893

Burton, M. E., Crooker, N. U., Siscoe, G. L., \& Smith, E. J. 1994, Journal of Geophysical Research, 99, 1

Case, A. W., Kasper, J. C., Stevens, M. L., et al. 2020, The Astrophysical Journal Supplement Series, 246, 43 
Chen, C. H. K., Chandran, B. D. G., Woodham, L. D., et al. 2021 [arXiv: 2101.00246]

Crooker, N. U., Appleton, E. M., Schwadron, N. A., \& Owens, M. J. 2010, Journal of Geophysical Research: Space Physics, 115

Crooker, N. U. \& Intriligator, D. S. 1996, Journal of Geophysical Research: Space Physics, 101, 24343

Crooker, N. U., Kahler, S. W., Gosling, J. T., \& Lepping, R. P. 2008, Journal of Geophysical Research: Space Physics, 113, 1

Crooker, N. U., McAllister, A. H., Fitzenreiter, R. J., et al. 1998, Journal of Geophysical Research: Space Physics, 103, 26859

Crooker, N. U., Siscoe, G. L., Shodhan, S., et al. 1993, Journal of Geophysical Research, 98, 9371

Desai, R. T., Zhang, H., Davies, E. E., et al. 2020, Solar Physics, 295, 130

Feldman, W. C., Asbridge, J. R., Bame, S. J., Montgomery, M. D., \& Gary, S. P. 1975, Journal of Geophysical Research (1896-1977), 80, 4181

Forsyth, R. J., Balogh, A., Smith, E. J., \& Gosling, J. T. 1997, Geophysical Research Letters, 24, 3101

Fox, N. J., Velli, M. C., Bale, S. D., et al. 2016, Space Science Reviews, 204, 7

Galvin, A. B., Kistler, L. M., Popecki, M. A., et al. 2008, Space Science Reviews, 136,437

Glassmeier, K.-H., Auster, H.-U., Heyner, D., et al. 2010, Planetary and Space Science, 58,287

Gosling, J. T., Baker, D. N., Bame, S. J., et al. 1987, Journal of Geophysical Research, 92, 8519

Gosling, J. T., Bame, S. J., Mccomas, D. J., et al. 1993, Geophysical Research Letters, 20, 2789

Gosling, J. T., Birn, J., \& Hesse, M. 1995, Geophysical Research Letters, 22, 869

Gosling, J. T., Borrini, G., Asbridge, J. R., et al. 1981, Journal of Geophysical Research, 86, 5438

Gosling, J. T. \& Pizzo, V. J. 1999, Space Science Reviews, 89, 21

Gosling, J. T., Skoug, R. M., \& Feldman, W. C. 2001, Geophysical Research Letters, 28, 4155

Heyner, D., Richter, I., Plaschke, F., et al. 2020, in EGU General Assembly 2020

Hickmann, K. S., Godinez, H. C., Henney, C. J., \& Arge, C. N. 2015, Solar Physics, 290, 1105

Hoeksema, J. T. 1991, Advances in Space Research, 11, 15

Hoeksema, J. T., Wilcox, J. M., \& Scherrer, P. H. 1983, Journal of Geophysical Research, 88, 9910

Horbury, T. S., O’Brien, H., Carrasco Blazquez, I., et al. 2020, A\&A, 642, A9

Jian, L., Russell, C. T., Luhmann, J. G., \& Skoug, R. M. 2006, Solar Physics, 239, 337

Jian, L. K., MacNeice, P. J., Taktakishvili, A., et al. 2015, Space Weather, 13, 316

Jones, G. H., Afghan, Q., \& Price, O. 2020, Research Notes of the $\{$ AAS \}, 4, 62

Jones, G. H. \& Balogh, A. 2000, Journal of Geophysical Research: Space Physics, 105, 12713

Kaiser, M. L., Kucera, T. A., Davila, J. M., et al. 2008, Space Science Reviews, 136,5

Kasper, J. C., Abiad, R., Austin, G., et al. 2016, Space Science Reviews, 204, 131

Kilpua, E., Koskinen, H. E. J., \& Pulkkinen, T. I. 2017, Living Reviews in Solar Physics, 14, 5

Klein, L. \& Burlaga, L. 1980, Journal of Geophysical Research, 85, 2269

Kruse, M., Heidrich-Meisner, V., \& Wimmer-Schweingruber, R. F. 2021, A\&A 645, A83

Lee, M. A. 2000, Journal of Geophysical Research: Space Physics, 105, 10491

Lepping, R. P., Acũna, M. H., Burlaga, L. F., et al. 1995, Space Science Reviews, 71, 207

Lin, R. P., Anderson, K. A., Ashford, S., et al. 1995, Space Science Reviews, 71, 125

Matteini, L., Laker, R., Woodham, L. D., et al. 2021, Astronomy \& Astrophysics, submitted

McComas, D. J., Gosling, J. T., Hammond, C. M., et al. 1994, Geophysical Research Letters, 21, 1751

McComas, D. J., Riley, P., Gosling, J. T., Balogh, A., \& Forsyth, R. 1998, Journal of Geophysical Research

Müller, D., Marsden, R. G., St. Cyr, O. C., \& Gilbert, H. R. 2013, Solar Physics, 285,25

Nakagawa, T., Nishida, A., \& Saito, T. 1989, Journal of Geophysical Research, 94, 11761

Nikolić, L. 2019, Space Weather, 17, 1293

Nolte, J. T. \& Roelof, E. C. 1973, Solar Physics, 33, 483

Odstrcil, D. 2003, Advances in Space Research, 32, 497

Ogilvie, K. W., Chornay, D. J., Fritzenreiter, R. J., et al. 1995, Space Science Reviews, 71, 55

Ogilvie, K. W. \& Desch, M. D. 1997, Advances in Space Research, 20, 559

Owen, C. J., Bruno, R., Livi, S., et al. 2020, A\&A, 642, A16

Owens, M., Lang, M., Barnard, L., et al. 2020a, Solar Physics, 295, 43

Owens, M. J., Crooker, N. U., \& Lockwood, M. 2013, Journal of Geophysical Research: Space Physics, 118, 1868
Owens, M. J., Lang, M., Riley, P., Lockwood, M., \& Lawless, A. S. 2020b, Journal of Space Weather and Space Climate, 10,8

Owens, M. J. \& Lockwood, M. 2012, Journal of Geophysical Research: Space Physics, 117, 1

Palmer, I. D., Allum, F. R., \& Singer, S. 1978, Journal of Geophysical Research: Space Physics, 83, 75

Panasenco, O., Velli, M., D’Amicis, R., et al. 2020, The Astrophysical Journal Supplement Series, 246, 54

Parker, E. N. 1958, The Astrophysical Journal, 128, 664

Peng, J., Liu, Y. C.-M., Huang, J., et al. 2017, Journal of Geophysical Research: Space Physics, 122, 9803

Pilipp, W. G., Miggenrieder, H., Montgomery, M. D., et al. 1987, Journal of Geophysical Research: Space Physics, 92, 1093

Pizzo, V. J. 1991, Journal of Geophysical Research: Space Physics, 96, 5405

Pizzo, V. J. 1994, Journal of Geophysical Research: Space Physics, 99, 4185

Pomoell, J. \& Poedts, S. 2018, J. Space Weather Space Clim., 8, A35

Richardson, I. G. 2018, Living Reviews in Solar Physics, 15, 1

Richardson, I. G. \& Cane, H. V. 2010, Solar Physics, 264, 189

Richardson, J. D. \& Paularena, K. I. 1997, Geophysical Research Letters, 24 1435

Richter, A. K. \& Luttrell, A. H. 1986, Journal of Geophysical Research: Space Physics, 91, 5873

Riley, P., Linker, J. A., Americo Gonzalez Esparza, J., et al. 2012, Journal of Atmospheric and Solar-Terrestrial Physics, 83, 11

Riley, P., Linker, J. A., \& Mikić, Z. 2002, Journal of Geophysical Research: Space Physics, 107, 1

Riley, P., Linker, J. A., Mikić, Z., et al. 2006, The Astrophysical Journal, 653, 1510

Robbrecht, E. \& Wang, Y.-M. 2012, The Astrophysical Journal, 755, 135

Rosenbauer, H., Schwenn, R., Marsch, E., et al. 1977, Journal of Geophysics Zeitschrift Geophysik, 42, 561

Rosenberg, R. L. \& Coleman, P. J. 1969, Journal of Geophysical Research, 74, 5611

Rouillard, A. P., Kouloumvakos, A., Vourlidas, A., et al. 2020, The Astrophysical Journal Supplement Series, 246

Roussev, I. I., Gombosi, T. I., Sokolov, I. V., et al. 2003, The Astrophysical Journal, 595, L57

Schatten, K. H., Wilcox, J. M., \& Ness, N. F. 1969, Solar Physics, 6, 442

Schwenn, R. 1990, Large-Scale Structure of the Interplanetary Medium, ed. R. Schwenn \& E. Marsch (Berlin, Heidelberg: Springer Berlin Heidelberg), 99-181

Schwenn, R., Montgomery, M. D., Rosenbauer, H., et al. 1978, Journal of Geophysical Research: Space Physics, 83, 1011

Siscoe, G. L., Goldstein, B., \& Lazarus, A. J. 1969, Journal of Geophysical Research: Space Physics, 74

Smith, E. J. 2001, Journal of Geophysical Research: Space Physics, 106, 15819

Smith, E. J. \& Thomas, B. T. 1986, Journal of Geophysical Research: Space Physics, 91, 2933

Smith, E. J. \& Wolfe, J. H. 1976, Geophysical Research Letters, 3, 137

Stansby, D., Horbury, T. S., \& Matteini, L. 2019a, Monthly Notices of the Royal Astronomical Society, 482, 1706

Stansby, D., Horbury, T. S., Wallace, S., \& Arge, C. N. 2019b, Research Notes of the AAS, 3, 57

Stansby, D., Rai, Y., Argall, M., et al. 2020a, heliopython/heliopy: HelioPy 0.13 .0

Stansby, D., Yeates, A., \& Badman, S. T. 2020b, Journal of Open Source Software, 5,2732

Steiger, C., Montagnon, E., Accomazzo, A., \& Ferri, P. 2020, Acta Astronautica, 170,472

Suess, S. T., McComas, D. J., \& Hoeksema, J. T. 1993, Geophysical Research Letters, 20, 161

Szabo, A., Larson, D., Whittlesey, P., et al. 2020, The Astrophysical Journal Supplement Series, 246, 47

Telloni, D., Scolini, C., Mostl, C., et al. 2021, Astronomy \& Astrophysics, submitted

Temmer, M., Reiss, M. A., Nikolic, L., Hofmeister, S. J., \& Veronig, A. M. 2017, The Astrophysical Journal, 835, 141

Thomas, B. T. \& Smith, E. J. 1981, Journal of Geophysical Research, 86, 11105

Tsurutani, B. T., Gonzalez, W. D., Gonzalez, A. L. C., et al. 2006, Journal of Geophysical Research: Space Physics, 111

Villante, U., Bruno, R., Mariani, F., Burlaga, L. F., \& Ness, N. F. 1979, Journal of Geophysical Research: Space Physics, 84, 6641

Wang, Y.-M., N. R. Sheeley, J., Howard, R. A., et al. 1997, The Astrophysical Journal, 485, 875

Whittlesey, P. L., Larson, D. E., Kasper, J. C., et al. 2020, The Astrophysical Journal Supplement Series, 246, 74

Wilcox, J. M. \& Ness, N. F. 1965, Journal of Geophysical Research, 70, 5793

Yeates, A. 2018

Zhao, X. \& Hoeksema, J. T. 1996, Journal of Geophysical Research: Space Physics, 101, 4825

Zhao, X.-P. \& Hundhausen, A. J. 1981, Journal of Geophysical Research, 86, 5423

Zurbuchen, T. H. \& Richardson, I. G. 2006, In-Situ Solar Wind and Magnetic Field Signatures of Interplanetary Coronal Mass Ejections (New York, NY: Springer New York), 31-43 


\section{Appendix A: Additional Material}

\begin{tabular}{|c|c|c|c|c|c|c|c|c|c|}
\hline Event & Date & Type & $\begin{array}{l}\Phi \\
\left({ }^{\circ}\right)\end{array}$ & $\begin{array}{l}\Theta \\
\left({ }^{\circ}\right)\end{array}$ & $\begin{array}{c}R \\
\text { (AU) }\end{array}$ & $\begin{array}{l}\Phi_{S S} \\
\left({ }^{\circ}\right)\end{array}$ & $\begin{array}{l}\text { MVA Start } \\
\text { End }\end{array}$ & $\begin{array}{c}\operatorname{MVA}(\phi, \theta) \\
\left({ }^{\circ},{ }^{\circ}\right)\end{array}$ & $\lambda_{2} / \lambda_{3}$ \\
\hline SO_2005XN & 29/05/2020 00:00 & ICME & 290 & 3.9 & 0.56 & 330 & - & - & \\
\hline BC_2005OW & 29/05/2020 15:30 & $\begin{array}{l}\mathrm{HCS} \\
\mathrm{CIR}\end{array}$ & 252 & -2.7 & 0.97 & 325 & $\begin{array}{l}29 / 05 / 2020 \\
30.05 / 2020 \\
08: 20\end{array}$ & $(31,23)$ & 18 \\
\hline Wind_2005 AW & 30/05/2020 04:18 & $\begin{array}{l}\mathrm{HCS} \\
\mathrm{CIR}\end{array}$ & 250 & -0.9 & 1.01 & 320 & $\begin{array}{lll}29 / 05 / 2020 & 15: 51 \\
30 / 05 / 2020 & 15: 51\end{array}$ & $(27,18)$ & 13 \\
\hline BC_2006LP & $06 / 06 / 202018: 30$ & $\begin{array}{l}\mathrm{HCS} \\
\mathrm{CIR}\end{array}$ & 140 & -1.9 & 0.95 & 198 & $\begin{array}{l}\text { 07/06/2020 01:50 } \\
07 / 06 / 202013: 50\end{array}$ & $(17,-31)$ & 5.2 \\
\hline Wind_2006HR & 07/06/2020 12:00 & $\begin{array}{l}\mathrm{HCS} \\
\mathrm{CIR}\end{array}$ & 140 & 0.1 & 1.01 & 216 & $\begin{array}{l}\text { 07/06/2020 03:48 } \\
\text { 08/06/2020 03:48 }\end{array}$ & $(28,-26)$ & 5.9 \\
\hline SO_2006ZS & 07/06/2020 18:00 & \begin{tabular}{|l} 
HCS \\
CIR \\
ICMES
\end{tabular} & 184 & 5.9 & 0.53 & 220 & $\begin{array}{l}07 / 06 / 202016: 10 \\
08 / 06 / 202016: 10\end{array}$ & $(20,-18)$ & 11 \\
\hline BC_2006YZ & 22/06/2020 21:00 & HCS & 260 & -0.2 & 0.9 & 375 & $\begin{array}{l}22 / 06 / 202014: 30 \\
23 / 06 / 202002: 30\end{array}$ & $(31,-16)$ & 10.5 \\
\hline BC_2006YU & 25/06/2020 14:00 & $\begin{array}{l}\mathrm{HCS} \\
\mathrm{CIR}\end{array}$ & 295 & 0.1 & 0.89 & 325 & $\begin{array}{lll}25 / 06 / 2020 & 10: 00 \\
25 / 06 / 2020 & 22: 00\end{array}$ & $(33,32)$ & 17.5 \\
\hline PSP_2006XO & 26/06/2020 00:00 & ICME & 280 & 2.4 & 0.52 & 316 & - & - & \\
\hline Wind_2006JJ & 26/06/2020 13:00 & $\begin{array}{l}\mathrm{HCS} \\
\mathrm{CIR}\end{array}$ & 241 & 2.4 & 1.02 & 330 & $\begin{array}{l}\text { 26/06/2020 13:00 } \\
27 / 06 / 202013: 00\end{array}$ & $(34,26)$ & 10.2 \\
\hline SO_2007KG & 02/07/2020 04:00 & HCS & 270 & 5 & 0.56 & 310 & $\begin{array}{l}\text { 01/07/2020 22:00 } \\
02 / 07 / 202010: 00\end{array}$ & $(20,-32)$ & 6.1 \\
\hline Wind_2007BU & 02/07/2020 11:00 & $\begin{array}{l}\mathrm{HCS} \\
\mathrm{CIR}\end{array}$ & 170 & 3 & 1.02 & 250 & $\begin{array}{l}\text { 02/07/2020 14:05 } \\
\text { 03/07/2020 02:05 }\end{array}$ & $(41,-52)$ & 7.2 \\
\hline BC_2007JH & 03/07/2020 23:00 & $\begin{array}{l}\mathrm{HCS} \\
\mathrm{CIR}\end{array}$ & 150 & 1.1 & 0.86 & 210 & $\begin{array}{l}03 / 07 / 202017: 50 \\
03 / 07 / 2020 \text { 23:50 }\end{array}$ & $(57,-34)$ & 6 \\
\hline
\end{tabular}

Table A.1. List of events with significant MVA results used in this paper. The string before the underscore denotes the spacecraft that made the measurement; the digits represent the two digits year and month with the final two random characters differentiating the events within each month. MVA was used to determine the orientation of the boundary, and is left blank for CMEs.

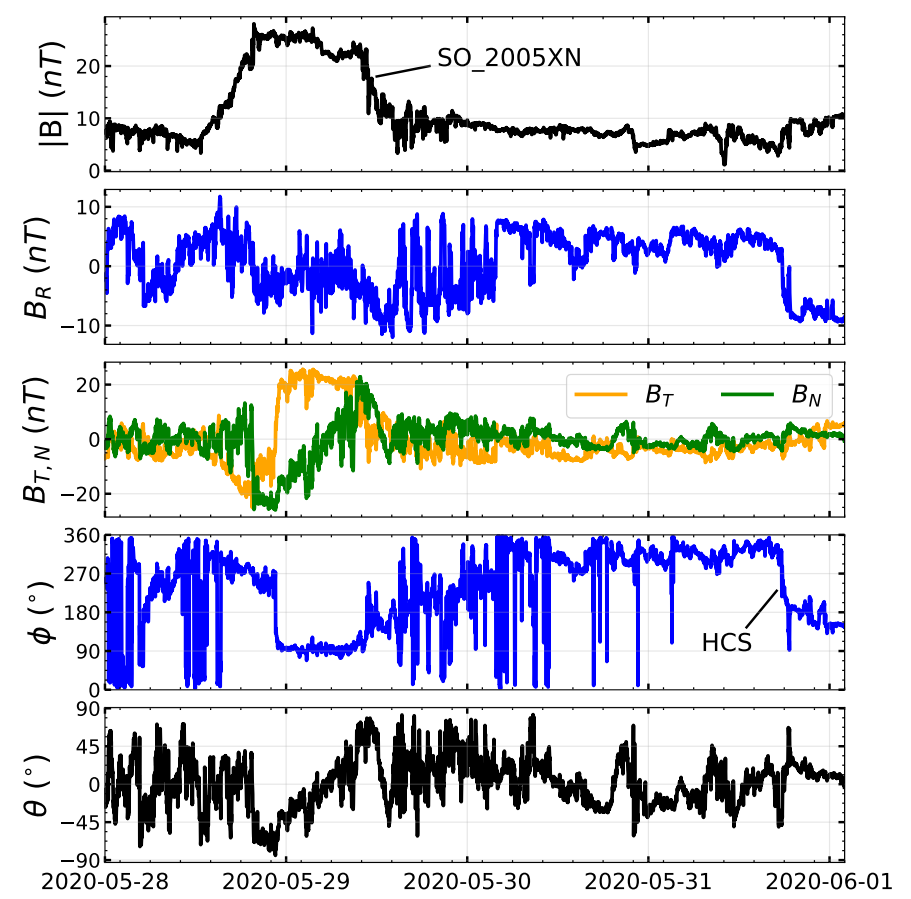

Fig. A.1. Transient feature, SO_2005XN, seen by SO during CR2231. There is a smooth rotation in the magnetic field, indicating a flux rope with radial minimum variance direction. PAS measurements indicate that the sign of $\sigma_{C}$ reverses across the boundary marked as a HCS crossing. A similar flux rope structure was observed on the next solar rotation by PSP, Fig. 4, at the same distance and longitude. Like the PSP observations, there is a reversal in the field, although this occurs $\sim 3$ days after the flux rope in this case. 